\title{
La Mapoteca Virtual de la Universidad Nacional de Costa Rica
}

\author{
Adolfo Quesada Román*
}

\section{Resumen}

La Mapoteca Virtual de la Universidad Nacional de Costa Rica es una actividad permanente de extensión que busca promover el uso de las TIC (Tecnologías de la información y la comunicación), así como de las TIG (Tecnologías de la información geográfica) en Costa Rica. Sus funciones están concentradas en un sitio web que se exhibe a través de la ideología del Open Access (Acceso Abierto), el cual cuenta con una serie de productos de cartografía temática, manuales sobre cartografía, recursos electrónicos y publicaciones relacionadas a las Ciencias Geográficas y otras afines.

\section{Palabras clave}

Mapoteca Virtual, Cartografía, Geografía, Historia, Geografía Histórica, Costa Rica, Universidad Nacional

\begin{abstract}
The Virtual Map Library of the National University of Costa Rica it's a permanent connection activity that looks to promote the use of ICT (Information and communications technology) and GIT (Geographic information technology) in Costa Rica. Its functions are concentrated in a website that's exhibits through an Open Access ideology a series of products of thematic cartography, manuals about cartography, electronic resources and publications related to the Geographical Sciences and others related.
\end{abstract}

\section{Keywords}

Virtual Map Library, Cartography, Geography, History, Historical Geography, Costa Rica, National University 


\section{Introducción}

La representación del espacio se realiza por medio de mapas, los cuales conforme pasa el tiempo, van perfeccionado sus técnicas y precisión. El conocimiento del espacio ha sido fundamental en el desarrollo de la civilización, sus implicaciones han sido estratégicas tanto para ubicarse por medio de las estrellas como para localizar puntos de caza durante las grandes migraciones. Los mapas tienen múltiples funciones, y detrás de su confección hay una connotación geopolítica o socioeconómica.

Durante el periodo de conquista y colonización de América, por ejemplo, el conocimiento de las costas, la ubicación de asentamientos indígenas, de recursos mineros, de cursos fluviales, fueron elementos centrales que impulsaron la cartografía de la época, por parte de la corona española. Es por ello, que se tiene un acervo importante de cartografía político-administrativa desde el siglo XVI, en el cual han quedado representados los países del área en sus diferentes etapas históricas.

Todos estos documentos tienen un valor histórico vital para la comprensión de procesos sociales, económicos e incluso ambientales. Los documentos deben ser escaneados para ser utilizados en la Mapoteca Virtual, el tamaño y la calidad de los documentos originales varía; por tanto, la determinación de los ajustes a la hora de los escaneos de la información, para su posterior manejo y descarga sin mayores complicaciones desde cualquier navegador, resulta en todo un reto, como también lo ha sido en otros proyectos en el país, como la Biblioteca Virtual en Población y Salud del Centro Centroamericano de Población (Chinchilla-Arley, 2011).

La digitalización de bases de datos cartográficas es una prioridad en muchas bibliotecas de la región latinoamericana como en México, donde por ejemplo la mapoteca del Centro de Conocimiento del Agua (Cenca) cuenta aproximadamente con 8,500 cartas, mapas y planos generados principalmente por el Instituto Nacional de Estadística, Geografía e Informática (INEGI), y sus antecesores como la Comisión de Estudios del Territorio Nacional (Cetenal), se encuentra en un proceso de digitalización de todo su acervo histórico por medio de una sistematización que requiere un orden estricto en el llenado de los metadatos, en los cuales se definieron las entidades (mapas) y sus atributos (características), así como las relaciones que existen entre ellas. La catalogación e indización de material cartográfico exige, 
además de los conocimientos propios de la profesión bibliotecológica, tener conocimientos básicos de cartografía. En síntesis, es necesario familiarizarse con este tipo de materiales para catalogarlos, clasificarlos, indizarlos, además de aprender a preservarlos y difundirlos (Navarro, Vargas y Noriega, 2014).

Otro ejemplo es la realización de una cartoteca digital al norte de Brasil, en la que existía una dificultad del acceso libre a las bases cartográficas digitales, ya que había altos costes financieros, el alto grado de sofisticación tecnológica y el tiempo que se necesita para estos trabajos. Además, en un país de las dimensiones de Brasil, estas dificultades se ven potencializadas por la gran extensión del territorio. El objetivo de la cartoteca digital es democratizar el uso de las bases cartográficas digitales, para su uso en los diversos campos de la geografía y de las ciencias afines.

Estos archivos se crean en diversos programas (software) y tienen diversas arquitecturas internas, expresadas con su correspondiente extensión. Dada la gran cantidad y diversidad de tipos de archivos generados, es necesario la creación de rutinas y procedimientos de búsqueda para su localización en función del tipo. Para 2004, la base de datos de la Cartoteca Digital Humboldt, contaba con más de 1.000 archivos, cubriendo principalmente el Estado de Paraíba y el noreste brasileño (Vianna y Neves, 2004).

Los repositorios de acceso abierto pueden ser de dos tipos: institucionales o especializados, los cuales son archivos digitales que pretenden recoger toda la producción científica de un país, una institución o una región en un sitio web que esté a disposición de los usuarios en forma gratuita y sin restricciones; por lo que, deben ser interoperables para que la información contenida pueda ser intercambiable entre las diferentes colecciones existentes. No existe en Costa Rica ningún mandato que obligue a los académicos a depositar sus producciones en los repositorios existentes, ni invitaciones que inciten al autoarchivo de la producción en determinadas instituciones. Tampoco existen propuestas al respecto, las únicas iniciativas tienden a ejecutar solo la recopilación de textos, principalmente por parte de sus bibliotecas, y por ello, todos los repositorios encontrados contienen pocos documentos, son de formación reciente, no incluyen normas para compartir los metadatos o lograr su interoperabilidad y 
pueden considerarse incompletos; no obstante, vale resaltar que las universidades públicas han sido las organizaciones que han desarrollado más repositorios de información (Córdoba-González, 2011).

Efectivamente, los repositorios se han convertido en las bibliotecas del siglo XXI y han llenado un espacio que anteriormente era impensable para los países con escaso desarrollo. Es fundamental delinear una política institucional o nacional para formarlos, pero también requiere articular la formación de los profesionales involucrados en su desarrollo, para crear conciencia sobre su importancia, y así actuar voluntariamente depositando sus artículos en los repositorios existentes y alimentarlos sin esperar ninguna recompensa material (Córdoba-González, 2011).

\section{La Mapoteca Virtual y su historia}

Durante el año 2007, el académico e investigador de la Escuela de Ciencias Geográficas de la Universidad Nacional de Costa Rica (UNA), Gustavo Barrantes Castillo propuso mediante un proyecto de investigación y posterior actividad permanente, el desarrollo de un sitio web denominado la Mapoteca Virtual, el cual se fundamenta como una plataforma de software libre hecha para almacenar mapas digitales en distintos formatos (TIFF, JPEG, SHP y otros), así como diversas cartografías y publicaciones relacionadas con las Ciencias de la Tierra, el Ordenamiento Territorial, la Gestión del Riesgo de Desastres y los Recursos Naturales en su mayoría de Costa Rica (Barrantes, Tercero y Morera, 2010).

El espíritu de esta iniciativa siempre ha sido poner a disposición del público en general (estudiantes, profesores y ciudadanos de todos los niveles educativos) una amplia colección mapas digitales producidos por diversas instituciones públicas y especialistas de la UNA, puesto que la oferta aumenta gracias a manuales sobre técnicas cartográficas y de uso de Sistemas de Información Geográfica (SIG), y una muestra de productos académicos relacionados con la cartografía y el software libre relacionado con los SIG. 
En términos generales, los objetivos de esta iniciativa son (Barrantes, 2011):

- Proveer material digital que permita la incorporación de las Tecnologías de la Información y la Comunicación (TIC) en el proceso de enseñanza y aprendizaje.

- Acercar a los investigadores por medio de la difusión de sus productos cartográficos.

- Facilitar el uso y acceso libre de materiales relacionados con la cartografía y la geografía a estudiantes de primaria y secundaria, universitarios y para el público en general.

La página de la Mapoteca Virtual está basada en el Sistema Gestor de Contenido o CMS (Content Management System por sus siglas en inglés,) Joomla; el cual es una plataforma de software que permite la subida y administración de contenidos dirigidos a sitios web. Entre sus ventajas están que es gratuito y cuenta consoporte técnico mediante blogs en línea. Además tiene una interfaz de administración fácil de utilizar, lo que permite que cualquier persona pueda administrar sus contenidos web sin la necesidad de poseer conocimientos técnicos especializados (Barrantes, Tercero, Picado y Morera, 2010). La Mapoteca Virtual de la UNA puede accederse por medio del sitio: http://www.mapoteca.geo.una.ac.cr/ y consta de una amplia colección de cartografía que ha sido clasificada de acuerdo a la temática ad hoc a sus contenidos (Fig. 1).

Figura 1. Página principal del sitio de Mapoteca Virtual (junio, 2015)

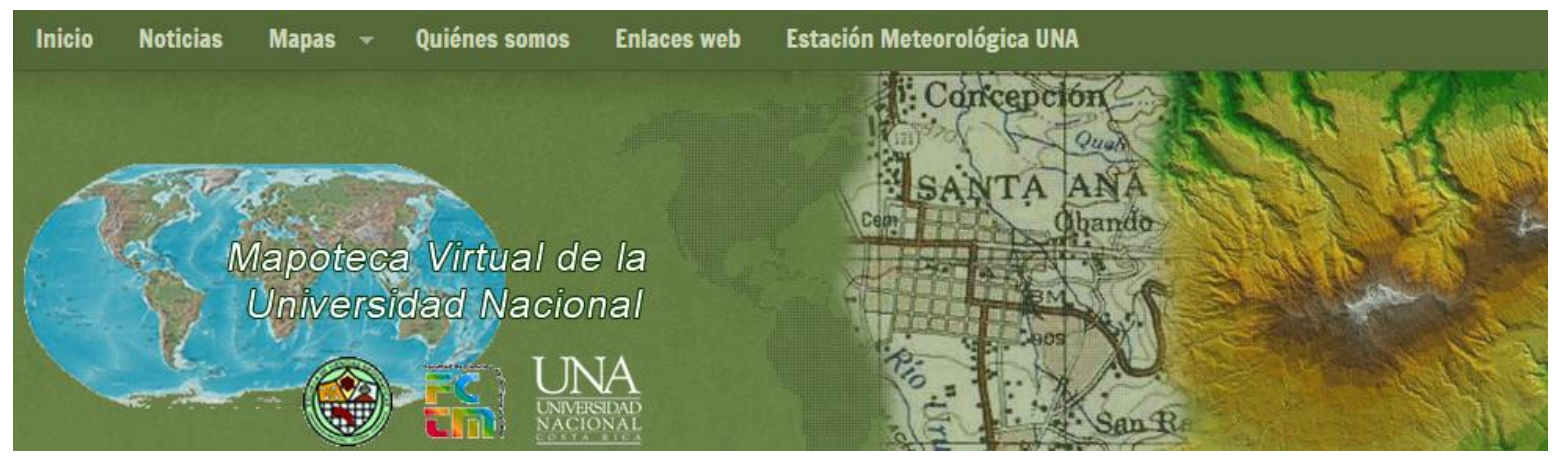

Dichas categorías conforman un total de 511 mapas que están disponibles para el público en general, además esta colección está en constante aumento debido al cordial apoyo de muchos investigadores y académicos de la UNA, así como otras instituciones académicas y gubernamentales del país que facilitan sus cartografías para ser compartidas dentro de la filosofía del Acceso Directo (Open Access). 
Cabe resaltar que las categorías de Amenazas y Riesgos y Uso del Suelo son las que mayor cantidad de insumos cartográficos poseen, donde el 63,7\% del total de mapas se concentran en estas dos clases.

\section{Los alcances de la Mapoteca Virtual}

A lo largo de su historia, la Mapoteca Virtual se ha posicionado en la oferta nacional y regional como una página Web, con una variedad amplia de cartografía que tiene como intención ser de utilidad para la investigación científica, la enseñanza y el conocimiento en general. Para 2015, se ofrece una sección superior inicial donde se disponen los siguientes accesos: Inicio, Noticias, Mapas, Quienes somos, Enlaces Web y Estación Meteorológica UNA.

En la pantalla de Inicio se destacan todos los elementos incluidos de manera reciente, además todos los distintos accesos a información variada que tiene la página como los diferentes portales de cartografía digital del país, los que no tienen relación directa con la UNA, entre ellas están los Temas clave, los cuales a su vez se subdividen en Sistemas de Información Geográfica, Software Libre, Cartografía, Geografía, Recursos y todos los blogs. En la sección Noticias, la intención es publicar las diversas novedades respecto a cartografía digital en la UNA y el país, no obstante en la actualidad se encuentra desactualizada.

En la sección de Mapas, se concentran 16 clases: Amenazas y riesgos, Cartografía Base, Clima, Contaminación ambiental, Economía y desarrollo, Fotografías aéreas, Geología, Geomorfología, Hidrografía, Imágenes satelitales, Líneas vitales, Población, Suelo, Transportes, Uso del suelo y Vegetación y ecología. En el apartado Quienes somos simplemente se muestran las personas a quienes contactar en caso de alguna duda o búsqueda de información precisa (ver Fig. 2). 
Figura 2. Temáticas de la Cartografía de la Mapoteca Virtual de la UNA

\begin{tabular}{|c|c|c|c|}
\hline Amenazas y riesgos & Economía y desarrollo & Hidrografía & Suelo \\
\hline Cartografía Base & Fotografías aéreas & Imágenes satelitales & Transportes \\
\hline Clima & Geología & Líneas vitales & Uso del suelo \\
\hline Contaminación ambiental & Geomorfología & Población & Vegetación y ecología \\
\hline
\end{tabular}

Elaboración propia del autor

En el apartado Enlaces Web la información recabada se divide en Cartografía y Geografía; el primero, por un lado, consta de 12 publicaciones que se limitan a trabajos tanto de la UNA como externos, en los cuales intervienen productos cartográficos. Por otro lado, en la sección Geografía se tienen 21 estudios que constan de cartografías temáticas y análisis puntuales de las materias tratadas. Por último, en la sección Estación Meteorológica UNA se publica desde Febrero de 2012 y de manera continua desde Octubre de 2014, boletines mensuales de las condiciones meteorológicas en la Universidad Nacional de Costa Rica en el Campus Omar Dengo de Heredia, lo que permite ser una base para estudios multidisciplinarios y conocer los cambios en el clima local.

Hasta hoy (junio de 2015), la Mapoteca Virtual ha sido visitada en al menos 662000 veces, según sus registros. Cabe resaltar que existen ciertas publicaciones como manuales introductorios a la temática cartográfica y de uso de Sistemas de Información Geográfica que tienen un particular impacto en el número de visitas (Fig. 3).

Otro de los alcances tangibles de la Mapoteca Virtual ha sido el convenio específico entre la UNA (Universidad Nacional de Costa Rica) y de la DGAN (Dirección General del Archivo Nacional) firmado en 2013. Dicho acuerdo contempla la facilitación de un escáner de alta resolución por parte de la Escuela de Ciencias Geográficas, así como estudiantes en calidad de asistentes académicos, con el fin de escanear los mapas y planos antiguos; a cambio, el Área de Conservación del Archivo Nacional se comprometió a brindar un espacio de trabajo y la colección de mapas y planos. 
Figura 3. Artículos populares, momento de creación y número de visitas de la página de la Mapoteca Virtual de la UNA

\begin{tabular}{|c|c|}
\hline Artículos populares & Impresiones \\
\hline Introducción a las escalas & 120369 \\
\hline Como obtener coordenadas geográficas en una hoja 1:50.000 & 92150 \\
\hline Buscar descargar & 79156 \\
\hline Obtención de coordenadas Lambert & 43055 \\
\hline Buscar mapas & 34110 \\
\hline Mara un perfil topográfico & 31490 \\
\hline Mapas 1:50 000 de Costa Rica & 20761 \\
\hline Equipo de trabajo & 20339 \\
\hline CRTM05 y cambio de proyección & 16809 \\
\hline
\end{tabular}

Elaboración propia del autor

La importancia de proteger este acervo cultural, a la vez que hacerlo accesible a investigadores, estudiantes y amantes de la cartografía, ha llevado al desarrollo de una Cartoteca Histórica de Costa Rica y un marco de colaboración entre las dos Instituciones. Este vínculo es un gran paso en el desarrollo conjunto entre instituciones públicas del país, para integrar iniciativas que sean de beneficio para los dos entes interesados, la comunidad científica y el público en general. En el marco de este proyecto, se hace énfasis en la colaboración de estudiantes del Diplomado en Cartografía y Diseño Digital y del Bachillerato de Ciencias Geográficas de la Escuela de Ciencias Geográficas para realizar su práctica profesional a través del desarrollo de Atlas históricos temáticos.

El convenio entre la UNA y la DGAN ha permitido el rescate de la colección de mapas y planos que tienen un inestimable valor cultural, no solo para la historia nacional sino para la geopolítica y la geografía histórica. Los metadatos elaborados y su utilización como índices en los motores de búsqueda a nivel mundial, junto con la posibilidad que brinda el enfoque de Open Access, el cual permitirá que estos documentos puedan ser utilizados en una variedad de trabajos que van desde el ámbito escolar hasta la investigación científica. 
El desarrollo de atlas es una muestra del potencial que tiene la información levantada en este proyecto, tal es el caso del Atlas Histórico de Costa Rica: Límite Fronterizo con Nicaraguay el Atlas Histórico sobre las Bananeras de Costa Rica (Fig. 4), los cuales son un par de muestras del uso académico de esta información. Otros tipos de investigación que pueden derivarse de la colección de datos son estudios sobre el desarrollo de centros urbanos, cambios en el uso del suelo, problemas históricos en la disposición de desechos sólidos, la evolución en la red vial de Costa Rica, entre otros.

El convenio con el RONMAC (Red de Observación del Nivel del Mar de Costa Rica) del Departamento de Física de la Facultad de Ciencias Exactas y Naturales de la UNA, y la Mapoteca Virtual de la UNA maneja una estación meteorológica que proporciona datos meteorológicos cada 15 minutos. Desde Octubre de 2014, la publicación de boletines mensuales ha sido continua, en la que se constata su importancia y utilidad, al brindar datos mensuales; sino que también, se utiliza la información por horas para generar estudios de Química de la atmósfera, de biología y otras aplicaciones en trabajos finales de graduación de estudiantes así como insumos para la investigación científica.

\section{Figura 4. Encabezado título de los mapas hechos por la United Fruit Company en el caribe costarricense en 1902}

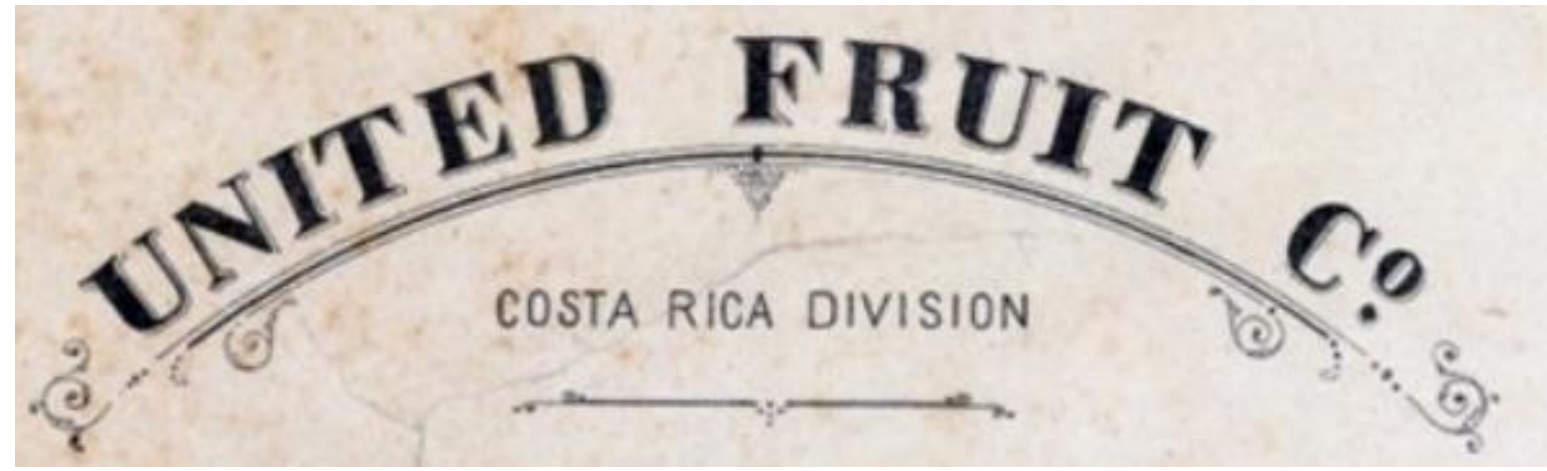

Fuente: Signatura 7186 del Archivo Nacional de Costa Rica.

A través del proyecto del Servicio de mapoteca virtual para la incorporación de las TIC en las actividades académicas, Código: 0198-15 del Sistema de Información Académica (SIA) de la UNA, se ha realizado varias publicaciones científicas como las de Barrantes et al., 2010 y Barrantes et al., 2011; 
en ambas, se mostró cómo este proyecto generaba información valiosa para la divulgación científica, el desarrollo de trabajos multidisciplinarios y como una plataforma única en su tipo en Costa Rica.

\section{Siempre se apunta al Norte}

La Mapoteca Virtual es una Actividad Permanente de Extensión avalada por la Facultad de Ciencias de la Tierra y el Mar de la UNA, y tiene una serie de actividades en un mediano plazo a ser desarrolladas. Uno de los logros a mediano plazo que el proyecto tiene contemplados está en la actualización de la plataforma, en la cual se basa la página de web del sitio, la cual actualmente se encuentra en Joomla 1.5 y quiere trasladarse la plataforma Joomla 3.0, dado que es software libre, su mejora continua es imperativa en un mundo digital cambiante.

Uno de los objetivos principales por los cuales se quiere modificar, en cuestiones de mejoras, la página de la Mapoteca Virtual radica en la forma en que los usuarios puedan acceder a la información que realmente les interesa. Además de hacer una serie de cambios en la interfaz gráfica con un aire más fresco, dinámico y accesible. Se quiere que la página tenga solamente tres grandes categorías: Cartografía (la cual se reorganizaría de 16 clases actuales, a unas 5 o 6 clases), Artículos (donde se incluirían manuales de cartografía básica, digital y otras publicaciones relativas al tema de los Sistemas de Información Geográfica generados en la ECG así como en la FCTM así como los boletines mensuales de la Estación Meteorológica) y Recursos (en este apartado se incluirían programas de software libre, páginas de interés científico y enlaces en general); esto se realizaría a la hora de renovar el sitio web de Joomla 1.5 a 3.0 .

Durante el 2015, se amplió el periodo de funcionamiento del proyecto de Mapoteca Virtual hasta 2019. Como una de las actividades que se quieren priorizar está la integración de diversos trabajos relacionados con cartografía digital que se realicen en la Facultad de Ciencias de la Tierra y Mar, esto ayudaría a ampliar significativamente la colección de mapas y trabajos realizados en la facultad, y en el acervo ya importante del sitio web, además tendría una importancia dual, ya que divulgaría la página así como el trabajo de los investigadores de la UNA. 
Uno de los temas que tiene una importancia transversal en el proyecto es el convenio que se tiene con el RONMAC (Red de Observación del Nivel del Mar de Costa Rica) del Departamento de Física de la Facultad de Ciencias Exactas y Naturales de la UNA, donde la Mapoteca Virtual administra una Estación Meteorológica y genera boletines mensuales, que facilitan información meteorológica a cada 15 minutos, para trabajos finales de graduación así como para investigaciones científicas. Uno de los objetivos de mejora para esta actividad es la divulgación en línea de esta información para que tenga un mayor impacto sobre los procesos que se presentan en la atmósfera en tiempo real.

El convenio que se tiene con el Archivo Nacional de Costa Rica traerá la publicación y divulgación de una Cartoteca Histórica (digitalización de mapas en papel para su protección histórica) por medio del Repositorio Institucional de la UNA, proceso que está en trámite con la Dirección de Tecnologías de Información y Comunicación (DTIC) de la UNA. De momento se tienen unos 1700 mapas escaneados que serán de gran utilidad para trabajos de geografía histórica, de historia y de otras ciencias afines.

La Mapoteca apunta a tener una mayor implementación en las labores de educadores de primaria y secundaria (en todos los ciclos educativos). Puesto que, busca ser el mejor sitio web de cartografía digital del país y América Central, por lo que al aumentar su divulgación es necesario por medio de redes sociales como Facebook, a su vez, se tratará de implementar un visualizador en línea de distintas capas de información cartográfica de Costa Rica por medio del servicio WMS (los cuales son datos referenciados espacialmente de forma dinámica a partir de información geográfica, es un estándar internacional para representar en forma de un archivo de imagen digital datos espaciales como calles, usos de la tierra, puntos de interés específicos y otros). 


\section{Conclusiones}

La Mapoteca Virtual de la UNA es el único sitio web en el país que concentra diversos productos de cartografía temática, manuales sobre cartografía, recursos electrónicos y artículos relacionados a las Ciencias Geográficas y otras afines en Costa Rica. Dada su popularidad entre los usuarios especializados, la intención de esta Actividad Permanente de la Escuela de Ciencias Geográficas de la UNA es la de ampliar de manera constante la colección de mapas. En primera instancia, en sincronización con las distintas unidades académicas (escuelas y centros de investigación) que pertenecen a la Facultad de las Ciencias de la Tierra y el Mar (FCTM) de la UNA, así como las demás dependencias de la Universidad, como por ejemplo la Escuela de Historia, y así con las carreras que enseñan Geografía y Estudios Sociales. Adicionalmente, será de vital relevancia integrar los esfuerzos cartográficos que se desarrollen en los entes gubernamentales y no gubernamentales del país con los derechos de autor del caso, para darles una mayor divulgación entre estudiantes, académicos y público en general.

La función de la Mapoteca Virtual aúna esfuerzos dentro de una línea de formación ciudadana a nivel global denominada geoalfabetización, la cual es la habilidad para utilizar la conceptualización y razonamiento geográfico en la toma de decisiones en todas las áreas del conocimiento (ciencias biológicas, químicas, de la salud, físico - matemáticas, ingenierías, sociales, humanidades y las artes) para el mejor entendimiento del sistema Tierra.

La intención primordial en un corto plazo es el de mejorar la plataforma visual de la página web mediante la actualización del Joomla, así como de aumentar considerablemente la colección actual de la cartografía temática generada por la UNA. En un mediano plazo, se quieren mejorar los lazos con las distintas dependencias de la UNA para divulgar los resultados cartográficos que la Universidad desarrolla en las distintas áreas del conocimiento (aquí se incluye la información de la Estación Meteorológica con el Departamento de Física). En un largo plazo, se quiere implementar un visualizador en línea de distintas capas de información cartográfica de Costa Rica por medio del servicio WMS. 


\section{Referencias}

Barrantes, G., Tercero, J., Picado, A. y Morera, C. (2010). La Mapoteca Virtual: un ejemplo de colaboración bajo una plataforma de software libre. Revista geográfica de América Central, $\mathrm{n}^{\circ}$ 45: 65-76.

Barrantes, G. (2011) Mapoteca Virtual: una herramienta tecnológica en apoyo a la educación. Revista geográfica de América Central, no 47: 1-10.

Brotton, J. (2014). Maps online: digital historical geographies. Journal of HistoricalGeography 43 (2014) 169-174.

Buzai, G. (2000). La exploración geodigital. Buenos Aires: Lugar Editorial.

Chinchilla-Arley, R. (2011). Biblioteca Virtual en Población y Salud del Centro Centroamericano de Población. E-Ciencias de la Información. Revista electrónica semestral, ISSN-1659-4142. Volumen 1, número 1, artículo 2. Enero - junio, 2011. Publicado 1 de enero, 2011. http://revistaebci.ucr.ac.cr/

Córdoba-Gonzaléz, S. (2011). Los repositorios institucionales y de acceso abierto en Costa Rica.EColabora: Revista de ciencia, educación, innovación y cultura apoyadas por Redes de Tecnología Avanzada, 2011, vol. 1, n. 2, pp. 86-100.

Navarro-Suástegui, P., Vargas-Suárez, V., Noriega-Luciano, C. (2014). Digitalización de la mapoteca: primera etapa. Informe final. Subcoordinación de Posgrado. Coordinación de Desarrollo Profesional e Institucional. DP-1429.1. Instituto Mexicano de Tecnología del Agua y la Secretaría de Medio Ambiente y Recursos Naturales (SEMARNAT).

Vianna, P., y Neves, I. (2004). Cartoteca Digital Humboldt. Geo Crítica / Scripta Nova. Revista electrónica de geografía y ciencias sociales. Barcelona: Universidad de Barcelona, 1 de agosto de 2004, vol. VIII, núm. 170-64. http://www.ub.es/geocrit/sn/sn-170-64.htm [ISSN: 1138-9788].

\section{Nota del Autor}

* Costarricense. Geógrafo y geomorfólogo. Egresado de la Maestría en Geografía Ambiental de la Universidad Nacional Autónoma de México (UNAM). Académico e Investigador de la Escuela de Ciencias Geográficas de la Universidad Nacional de Costa Rica (UNA). Correo electrónico: adolfo.quesada@gmail.com 